\title{
Pacific
}

Journal of

Mathematics

\section{Dec GROUPS FOR ARBITRARILY HIGH EXPONENTS}

BHARATH Al SETHURAmaN 


\title{
DEC GROUPS FOR ARBITRARILY HIGH EXPONENTS
}

\section{B. A. Sethuraman}

\begin{abstract}
For each prime $p$ and each $n \geq 1(n \geq 2$ if $p=2)$, examples are constructed of a Galois extension $K / F$ whose Galois group has exponent $p^{n}$ and a central simple $F$-algebra $A$ of exponent $p$ which is split by $K$ but is not in the Dec group of $K / F$.
\end{abstract}

1. Introduction. Let $K / F$ be an abelian Galois extension of fields, and let $G=\mathscr{G}(K / F)$. Let $G=G_{1} \times G_{2} \times \cdots \times G_{k}$ be a direct sum decomposition of $G$ into cyclic groups, with $G_{i}=\left\langle\sigma_{i}\right\rangle(i=1, \ldots, k)$. Let $F_{i}$ be the fixed field of $G_{1} \times \cdots \times G_{i-1} \times G_{i+1} \times \cdots \times G_{k}(i=$ $1, \ldots, k)$. Thus, the $F_{i}$ are cyclic Galois extensions of $F$, with Galois group isomorphic to $G_{i}$. The group $\operatorname{Dec}(K / F)$ is defined as the subgroup of $\operatorname{Br}(K / F)$ generated by the subgroups $\operatorname{Br}\left(F_{i} / F\right)(i=$ $1, \ldots, k)$. This group was introduced by Tignol ([T1]), where he shows that $\operatorname{Dec}(K / F)$ is independent of the choice of the direct sum decomposition of $G$. If $p$ is a prime, we will write $p^{n} \operatorname{Br}(K / F)$ and $p^{n} \operatorname{Dec}(K / F)$ for the subgroups of $\operatorname{Br}(K / F)$ and $\operatorname{Dec}(K / F)$ consisting of all elements with exponent dividing $p^{n}$.

A key issue in several past constructions of division algebras has been the non-triviality of the factor group ${ }_{p} \operatorname{Br}(K / F) /{ }_{p} \operatorname{Dec}(K / F)$ for suitable abelian extensions $K / F$. For instance, the Amitsur-RowenTignol construction of an algebra of index 8 with involution with no quaternion subalgebra ([ART]) depends crucially on the existence of a triquadratic extension $K / F$ for which ${ }_{2} \operatorname{Br}(K / F) \neq{ }_{2} \operatorname{Dec}(K / F)$. Similarly, the constructions of indecomposable algebras of exponent $p$ by Tignol ([T2]) and Jacob ([J]) also depend on the existence of an (elementary) abelian extension $K / F$ for which ${ }_{p} \operatorname{Br}(K / F) \neq{ }_{p} \operatorname{Dec}(K / F)$.

The extension fields $K / F$ that occur in these examples above are all of exponent $p$, and it is an interesting question whether there exist abelian extensions $K / F$ whose Galois groups have arbitrarily high ( $p$ power) exponents for which the factor group ${ }_{p} \operatorname{Br}(K / F) /{ }_{p} \operatorname{Dec}(K / F)$ is non-trivial. The purpose of this paper is to show that for each $n \geq 1(n \geq 2$ if $p=2)$, there exists an abelian extension $K / F$ with Galois group $\mathbb{Z} / p^{n} \mathbb{Z} \times \mathbb{Z} / p \mathbb{Z}$ (and thus, of exponent $p^{n}$ ) and an algebra $A \in{ }_{p} \operatorname{Br}(K / F)$ such that $A \notin{ }_{p} \operatorname{Dec}(K / F)$. (Note that if $K / F$ is an 
$\mathbb{Z} / 2 \times \mathbb{Z} / 2$ extension, then ${ }_{2} \operatorname{Br}(K / F)$ is always equal to ${ }_{2} \operatorname{Dec}(K / F)$, see [T3] for instance.)

Our field $F$ will be the rational function field in 3 variables over a field $F_{0}$ of characteristic 0 that contains sufficiently many roots of unity. (For instance, $F_{0}$ may be algebraically closed.) Our algebras will in fact be generalizations of the example given by Tignol in [T2]. Moreover, we will prove that for $A, K$, and $F$ as above, $A \otimes_{F} L \notin$ ${ }_{p} \operatorname{Dec}(K \cdot L / L)$ for any finite degree extension $L / F$ with $p \nmid[L: F]$.

The special case $n=2$ (and $p$ odd) of these computations was done in [Se1], where the result was used to construct non-elementary abelian crossed products of index $p^{3}$ and exponent $p^{2}$.

We remark that using different techniques, Rowen and Tignol ([RT]) have shown that if the ground field is assumed to only contain a primitive $p^{s}$ th root of unity but not a primitive $p^{s+1}$ th root of unity for some $s \geq 1$, then examples of non-trivial factor groups

$$
{ }_{p} \operatorname{Br}(K / F) / p \operatorname{Dec}(K / F)
$$

exist for suitable abelian extensions $K / F$ whose Galois groups have arbitrarily large ( $p$-power) exponents. Using ultraproducts $([\mathbf{R}])$, their example can be extended to also cover the case where the ground field contains all primitive $p^{i}$ th roots of unity $(i=1,2, \ldots)$.

2. $p$-adic valuations on rational function fields. Let $p$ be a prime, which, for now, can be either odd or even. Let $F_{0}$ be a field of characteristic 0 . The subfield $\mathbb{Q}$ of $F_{0}$ has a standard valuation $v: \mathbb{Q} \rightarrow \mathbb{Z}$ obtained by writing any non-zero element in $\mathbb{Q}$ as $p^{n} a / b$, where $n$, $a$, and $b$ are integers, and $p$ is relatively prime to $a$ and $b$, and defining $v\left(p^{n} a / b\right)=n$. We will refer to any valuation on $F_{0}$ that extends this distinguished valuation on $\mathbb{Q}$ as a $p$-adic valuation. Since the residue field of $\mathbb{Q}$ under $v$ is $\mathbb{Z} / p \mathbb{Z}$, the residue of $F_{0}$ under any $p$-adic valuation is of characteristic $p$.

Now let $F=F_{0}\left(x_{1}, x_{2}, \ldots, x_{k}\right)$ be the rational function field over $F_{0}$ in $k$ indeterminates $(k \geq 1)$, and let $v$ be a fixed $p$-adic valuation on $F_{0}$. Then $v$ admits an extension $w$ to $F$ defined as follows: for any polynomial $f \in F_{0}\left[x_{1}, x_{2}, \ldots, x_{k}\right], w(f)$ is the minimum of the values of the coefficients, and for $f$ and $g$ in $F_{0}\left[x_{1}, x_{2}, \ldots, x_{k}\right]$, $w(f / g)=w(f)-w(g)$. (It is easy to check that $w$ is indeed a valuation on $F$.) It can be shown that the residues $\bar{x}_{i}$ of the $x_{i}(i=1, \ldots, k)$ are algebraically independent over the residue $\bar{F}_{0}$ of $F_{0}$; and that, moreover, $\bar{F}$ is precisely the rational function field $\bar{F}_{0}\left(\overline{x_{1}}, \overline{x_{2}}, \ldots, \overline{x_{k}}\right)$. (It is also clear from the definition of $w$ that 
$\Gamma_{F}=\Gamma_{F_{0}}$.) We will refer to $w$ as the standard extension of $v$ to $F$. Also, we will abuse notation and continue to write $x_{i}$ for the residues $\bar{x}_{i}$.

REMARK 2.1. Furthermore, it can be shown that $w$ is the unique extension of $v$ to $F$ with the property that the values of the $x_{i}$ are 0 , and the residues of the $x_{i}$ are algebraically independent over $\overline{F_{0}}$. (See [B, §10, Proposition 2].)

The following is well known, but we include a proof here for convenience.

LEMMA 2.2. Let $p$ be any prime, and let $F$ be a field of characteristic 0 . Let $v$ be a p-adic valuation on $F$. Let $K=F\left(f^{1 / p}\right)$, where $f \notin F^{* p}$, and $v(f)=0$. Assume that $f=f_{0}^{p}+\pi f_{1}+\delta$, where $v\left(f_{0}\right)=v\left(f_{1}\right)=0,0<v(\pi)<(p /(p-1)) v(p)$, and $v(\delta)>v(\pi)$.

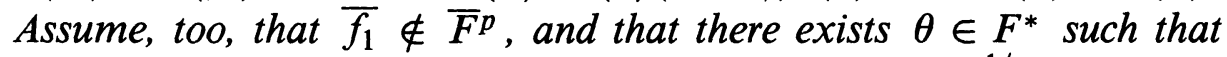
$\theta^{p}=\pi$. Then $v$ extends uniquely to $K$, and $\bar{K}=\bar{F}\left(\bar{f}_{1}^{1 / p}\right)$.

Proof. Let $r \in K^{*}$ satisfy $r^{p}=f$, and let $s=\left(r-f_{0}\right) / \theta$. Then $s+\left(f_{0} / \theta\right)=(r / \theta)$, so $s$ satisfies

$$
\left(s+\frac{f_{0}}{\theta}\right)^{p}=\frac{f_{0}^{p}+\pi f_{1}+\delta}{\theta^{p}} .
$$

Expanding the left-hand side of (1) and noting that $\theta^{p}=\pi$, we find

$$
s^{p}+\sum_{i=1}^{p-1}\left(\begin{array}{c}
p \\
i
\end{array}\right) s^{i}\left(\frac{f_{0}}{\theta}\right)^{p-i}=f_{1}+\left(\frac{\delta}{\theta^{p}}\right) .
$$

Now for $i=1, \ldots, p-1, v\left(\left(\begin{array}{l}p \\ i\end{array}\right)\right)=v(p)$, while $v\left(\theta^{p-i}\right) \leq v\left(\theta^{p-1}\right)$ $=v\left(\pi^{(p-1) / p}\right)<v(p)$. (The last inequality is because $v(\pi)<$ $(p /(p-1)) v(p)$.$) From this, as well as the fact that v\left(f_{0}\right)=0$, we find that each of the expressions $\left(\begin{array}{l}p \\ i\end{array}\right)\left(f_{0} / \theta\right)^{p-i}(i=1, \ldots, p-1)$ has positive value. It follows that for any extension $w$ of $v$ from $F$ to $K$, if $w(s)<0$, then the left-hand side of (2) would have the same value as $s^{p}$. (Here we use the fact that if $w(a)<w(b)$, then $w(a+b)=w(a)$.) Since this contradicts the fact that the value of the right-hand side of (2) is 0 (note that $v\left(f_{1}\right)=0$, while $v\left(\delta / \theta^{p}\right)>0$ ), we must have $w(s) \geq 0$. Similarly, if $w(s)>0$, then from $w(a+b) \geq \min (w(a), w(b))$, it follows that the left-hand side of (2) must have positive value. Hence $w(s)=0$. Taking the residues of each term in (2) and noting again that all terms except $s^{p}$ and $f_{1}$ have positive value, we find $\bar{s}^{p}=\bar{f}_{1}$. Thus $\bar{K} \supset \bar{F}\left(\bar{f}_{1}^{1 / p}\right)$. 
Since $\overline{f_{1}} \notin \bar{F}^{p}$, and since $[K: F]=p$, we find by the fundamental inequality ([E, Corollary 17.5$]$ ) that $w$ is unique, and $\bar{K}=$ $\bar{F}\left(\bar{f}_{1}^{1 / p}\right)$.

Now let $F_{0}$ be a field of characteristic 0 . We will assume that $F_{0}$ contains $p^{1 / p^{i}}$ for all $i(i=1,2, \ldots)$. Let $F$ be the rational function field $F_{0}\left(x_{1}, x_{2}, y\right)$. For each $n(n \geq 0)$, let

$$
\phi_{n}=\left(x_{1}^{p^{n}}-y^{p^{n}}\right)\left(x_{2}^{p^{n}}-y^{p^{n}}\right) .
$$

Let $H_{n}=F\left(\phi_{n}^{1 / p}\right)$. Let $v$ be the standard extension of any $p$-adic valuation on $F_{0}$ to $F$. The manner in which $v$ extends from $F$ to $H_{n}$ will be crucial to our Dec results, and the rest of $\S 2$ is devoted to this topic.

First, some notation. For $p$ odd, and $i=1,2, \ldots, p-1$, let

$$
\lambda_{i}=\frac{(-1)^{p-i}}{p}\left(\begin{array}{c}
p \\
i
\end{array}\right)
$$

(so each $\lambda_{i}$ is an integer). For $p$ odd, again, define $g_{n}(x, y) \in$ $\mathbb{Z}[x, y](n=0,1,2, \ldots)$ by

$$
g_{n}(x, y)=\sum_{i=1}^{p-1} \lambda_{i}\left(x^{p^{n}}\right)^{i}\left(y^{p^{n}}\right)^{p-i}
$$

so

$$
\left(x^{p^{n}}-y^{p^{n}}\right)^{p}=x^{p^{n+1}}-y^{p^{n+1}}+p g_{n}(x, y) .
$$

Now for $p$ odd, define $h_{n}\left(x_{1}, x_{2}, y\right) \in \mathbb{Z}\left[x_{1}, x_{2}, y\right](n=0,1,2, \ldots)$ by

(6) $h_{n}\left(x_{1}, x_{2}, y\right)=\left(x_{1}^{p^{n}}-y^{p^{n}}\right)^{p} g_{n}\left(x_{2}, y\right)+\left(x_{2}^{p^{n}}-y^{p^{n}}\right)^{p} g_{n}\left(x_{1}, y\right)$,

and for $p=2$, define $h_{n}\left(x_{1}, x_{2}, y\right) \in \mathbb{Z}\left[x_{1}, x_{2}, y\right](n=0,1,2, \ldots)$ by

$$
h_{n}\left(x_{1}, x_{2}, y\right)=\left(x_{1}^{2^{n}}+y^{2^{n}}\right)^{2} x_{2}^{2^{n}} y^{2^{n}}+\left(x_{2}^{2^{n}}+y^{2^{n}}\right)^{2} x_{1}^{2^{n}} y^{2^{n}} .
$$

REMARK 2.3. We will abuse notation and continue to write $g_{n}$ and $h_{n}$ for the images of $g_{n}$ and $h_{n}$ in $\mathbb{Z} / p \mathbb{Z}[x, y]$ and $\mathbb{Z} / p \mathbb{Z}\left[x_{1}, x_{2}, y\right]$ (respectively).

The special case $n=1$ (and $p$ odd) of the following was proved in [T2, Lemma 3.7]. 
Proposition 2.4. For every prime $p$ and for all $n(n \geq 1), v$ extends uniquely from $F$ to $H_{n}$, and $\overline{H_{n}}=\bar{F}\left(h_{0}\left(x_{1}, x_{2}, y\right)^{1 / p}\right)$.

Before proving Proposition 2.4, we need some further notation, as well as some easy lemmas.

For $p=2$, define $e_{n}\left(x_{1}, x_{2}, y\right) \in \mathbb{Z}\left[x_{1}, x_{2}, y\right]$ (for $n \geq 1$ ) by

$$
e_{n}\left(x_{1}, x_{2}, y\right)=y^{2^{n}}\left(x_{1}^{2^{n}}+x_{2}^{2^{n}}\right)
$$

and $\psi_{n}\left(x_{1}, x_{2}, y\right) \in \mathbb{Z}\left[x_{1}, x_{2}, y\right]$ (for $n \geq 0$ ) by

$$
\psi_{n}\left(x_{1}, x_{2}, y\right)=\left(x_{1}^{2^{n}}+y^{2^{n}}\right)\left(x_{2}^{2^{n}}+y^{2^{n}}\right)
$$

For $n \in \mathbb{Z}(n \geq 1)$, define $\alpha_{n} \in \mathbb{Q}$ by

$$
\alpha_{n}= \begin{cases}1, & \text { if } n=1 \\ 1+1 / p+1 / p^{2}+\cdots+1 / p^{n-1}, & \text { if } n>1\end{cases}
$$

Finally, for any $k \in \mathbb{Q}$, abbreviate the phrase "terms of value at least $v\left(p^{k}\right)$ " by $\left[\left[p^{k}\right]\right]$.

REMARK 2.5. Just as with $g_{n}$ and $h_{n}$, we will abuse notation and continue to write $e_{n}$ for the image of $e_{n}$ in $\mathbb{Z} / 2 \mathbb{Z}\left[x_{1}, x_{2}, y\right]$.

LEMMA 2.6. Let $f, g, f_{1}$, and $g_{1}$ be polynomials in $\mathbb{Z}\left[x_{1}, x_{2}, y\right]$. Then, with respect to the restriction of $v$ to $\mathbb{Q}\left(x_{1}, x_{2}, y\right)$ (i.e., the standard extension of the p-adic valuation on $\mathbb{Q}$ to $\left.\mathbb{Q}\left(x_{1}, x_{2}, y\right)\right)$,

1. If $f=g+[[p]]$, and $f_{1}=g_{1}+[[p]]$, then $f+f_{1}=g+g_{1}+[[p]]$ and $f f_{1}=g g_{1}+[[p]]$.

2. $(f+g)^{p}=f^{p}+g^{p}+[[p]]$.

3. Let $k \geq 1$, and suppose

$$
f=\sum c_{i_{1}, i_{2}, i_{3}}\left(x_{1}^{p^{k}}\right)^{i_{1}}\left(x_{2}^{p^{k}}\right)^{i_{2}}\left(x_{3}^{p^{k}}\right)^{i_{3}},
$$

for some $c_{i_{1}, i_{2}, i_{3}} \in \mathbb{Z}$. Define $f^{1 / p} \in \mathbb{Z}\left[x_{1}, x_{2}, y\right]$ by

$$
f^{1 / p}=\sum c_{i_{1}, i_{2}, i_{3}}\left(x_{1}^{p^{k-1}}\right)^{i_{1}}\left(x_{2}^{p^{k-1}}\right)^{i_{2}}\left(x_{3}^{p^{k-1}}\right)^{i_{3}} .
$$

Then $f=\left(f^{1 / p}\right)^{p}+[[p]]$.

Proof. Note that the values of $f, g, f_{1}$, and $g_{1}$ are non-negative. (1) and (2) are now elementary. (3) follows from (2) along with the fact that $a^{p} \equiv a(\bmod p)$ for any $a \in \mathbb{Z}$. 
LEMMA 2.7. With respect to the restriction of $v$ to $\mathbb{Q}\left(x_{1}, x_{2}, y\right)$,

1. For $n \geq 1$ and for all $p, h_{n}=h_{n-1}^{p}+[[p]]$, and for $n \geq 2$ and $p=2, e_{n}=e_{n-1}^{2}+[[2]]$.

2. For $n \geq 1$ and $p$ odd, $\phi_{n}=\phi_{n-1}^{p}-p h_{n-1}+\left[\left[p^{2}\right]\right]$.

3. For $n \geq 1$ and $p=2, \phi_{n}=\psi_{n}-2 e_{n}$, and $\psi_{n}=\psi_{n-1}^{2}-2 h_{n-1}+$ [[4]] (so $\left.\phi_{n}=\psi_{n-1}^{2}-2\left(h_{n-1}+e_{n}\right)+[[4]]\right)$.

Proof. (1) follows from the definitions of $h_{n}$ and $e_{n}$ and Lemma 2.6. For instance, for $p$ odd (and $n \geq 1$ ) we have

$$
\left(x_{1}^{p^{n}}-y^{p^{n}}\right)^{p}=\left(\left(x_{1}^{p^{n-1}}-y^{p^{n-1}}\right)^{p}+[[p]]\right)^{p}=\left(x_{1}^{p^{n-1}}-y^{p^{n-1}}\right)^{p^{2}}+[[p]] .
$$

Also,

$$
\begin{aligned}
g_{n}\left(x_{2}, y\right) & =\sum_{i=1}^{p-1} \lambda_{i}\left(x_{2}^{p^{n}}\right)^{i}\left(y^{p^{n}}\right)^{p-i} \\
& =\left(\sum_{i=1}^{p-1} \lambda_{i}\left(x_{2}^{p^{n-1}}\right)^{i}\left(y^{p^{n-1}}\right)^{p-i}\right)^{p}+[[p]] \\
& =\left(g_{n-1}\left(x_{2}, y\right)\right)^{p}+[[p]] .
\end{aligned}
$$

Since similar relations hold for $\left(x_{2}^{p^{n}}-y^{p^{n}}\right)^{p}$ and $g_{n}\left(x_{1}, y\right)$, we find

$$
\begin{aligned}
h_{n}= & \left(x_{1}^{p^{n-1}}-y^{p^{n-1}}\right)^{p^{2}}\left(g_{n-1}\left(x_{2}, y\right)\right)^{p} \\
& +\left(x_{2}^{p^{n-1}}-y^{p^{n-1}}\right)^{p^{2}}\left(g_{n-1}\left(x_{1}, y\right)\right)^{p}+[[p]] \\
= & \left(\left(x_{1}^{p^{n-1}}-y^{p^{n-1}}\right)^{p} g_{n-1}\left(x_{2}, y\right)\right. \\
& \left.\quad+\left(x_{2}^{p^{n-1}}-y^{p^{n-1}}\right)^{p} g_{n-1}\left(x_{1}, y\right)\right)^{p}+[[p]] \\
= & h_{n-1}^{p}+[[p]] .
\end{aligned}
$$

The proof for $p=2$ is similar. For (2), we have

$$
\begin{aligned}
\phi_{n}= & \left(x_{1}^{p^{n}}-y^{p^{n}}\right)\left(x_{2}^{p^{n}}-y^{p^{n}}\right) \\
= & {\left[\left(x_{1}^{p^{n-1}}-y^{p^{n-1}}\right)^{p}-p g_{n-1}\left(x_{1}, y\right)\right] } \\
& \cdot\left[\left(x_{2}^{p^{n-1}}-y^{p^{n-1}}\right)^{p}-p g_{n-1}\left(x_{2}, y\right)\right] \\
= & {\left[\left(x_{1}^{p^{n-1}}-y^{p^{n-1}}\right)\left(x_{2}^{p^{n-1}}-y^{p^{n-1}}\right)\right]^{p} } \\
& -p\left[\left(x_{1}^{p^{n-1}}-y^{p^{n-1}}\right)^{p} g_{n-1}\left(x_{2}, y\right)\right. \\
& \left.\quad+\left(x_{2}^{p^{n-1}}-y^{p^{n-1}}\right)^{p} g_{n-1}\left(x_{1}, y\right)\right]+\left[\left[p^{2}\right]\right] \\
= & \phi_{n-1}^{p}-p h_{n-1}+\left[\left[p^{2}\right]\right] .
\end{aligned}
$$


As for (3),

$$
\begin{aligned}
\phi_{n} & =\left(x_{1}^{2^{n}}-y^{2^{n}}\right)\left(x_{2}^{2^{n}}-y^{2^{n}}\right) \\
& =\left(x_{1}^{2^{n}}+y^{2^{n}}-2 y^{2^{n}}\right)\left(x_{2}^{2^{n}}+y^{2^{n}}-2 y^{2^{n}}\right) \\
& =\left(x_{1}^{2^{n}}+y^{2^{n}}\right)\left(x_{2}^{2^{n}}+y^{2^{n}}\right)-2 y^{2^{n}}\left(x_{1}^{2^{n}}+x_{2}^{2^{n}}\right) \\
& =\psi_{n}-2 e_{n} .
\end{aligned}
$$

Also,

$$
\begin{aligned}
\psi_{n}= & \left(x_{1}^{2^{n}}+y^{2^{n}}\right)\left(x_{2}^{2^{n}}+y^{2^{n}}\right) \\
= & {\left[\left(x_{1}^{2^{n-1}}+y^{2^{n-1}}\right)^{2}-2 x_{1}^{2^{n-1}} y^{2^{n-1}}\right]\left[\left(x_{2}^{2^{n-1}}+y^{2^{n-1}}\right)^{2}-2 x_{2}^{2^{n-1}} y^{2^{n-1}}\right] } \\
= & {\left[\left(x_{1}^{2^{n-1}}+y^{2^{n-1}}\right)\left(x_{2}^{2^{n-1}}+y^{2^{n-1}}\right)\right]^{2} } \\
& -2\left[\left(x_{1}^{2^{n-1}}+y^{2^{n-1}}\right)^{2} x_{2}^{2^{n-1}} y^{2^{n-1}}+\left(x_{2}^{2^{n-1}}+y^{2^{n-1}}\right)^{2} x_{1}^{2^{n-1}} y^{2^{n-1}}\right]+[[4]] \\
= & \psi_{n-1}^{2}-2 h_{n-1}+[[4]] .
\end{aligned}
$$

LEMMA 2.8. For all $p$ and for all $k \geq 0, \alpha_{k+1}<\alpha_{2}+1 / p$.

Proof. Since $\alpha_{1}<\alpha_{2}<\alpha_{2}+1 / p$, we may assume $k>2$. Now $\alpha_{k+1}=1+1 / p+\cdots+1 / p^{k}$ and $\alpha_{2}=1+1 / p$, so it is sufficient to prove that $1 / p^{2}+\cdots+1 / p^{k}<1 / p$. Multiplying both sides by $p$, we need to prove that $1 / p+\cdots+1 / p^{k-1}<1$. But this is clear, since

$$
\begin{aligned}
1 / p+\cdots+1 / p^{k-1} & =1 / p\left(1+1 / p+\cdots+1 / p^{k-2}\right) \\
& <1 / p\left(1+1 / p+1 / p^{2}+\cdots\right) \\
& =1 /(p-1) \leq 1
\end{aligned}
$$

Proof of Proposition 2.4. We divide the proof according to whether $p$ is odd or whether $p=2$.

Case 1 (Odd $p$ ). If $n=1$, this follows from Lemmas 2.7 and 2.2. For, by Lemma $2.7, \phi_{1}=\phi_{0}^{p}-p h_{0}+\delta$, for some $\delta \in \mathbb{Z}\left[x_{1}, x_{2}, y\right]$ with $v(\delta) \geq v\left(p^{2}\right)$. By assumption, $p^{1 / p} \in F_{0}$. Clearly, $-h_{0} \notin \bar{F}^{p}=$ $\bar{F}_{0}^{p}\left(x_{1}^{p}, x_{2}^{p}, y^{p}\right)$. Thus, by Lemma $2.2, v$ extends uniquely to $H_{1}$, and $\overline{H_{1}}=\bar{F}\left(\left(-h_{0}\right)^{1 / p}\right)=\bar{F}\left(h_{0}^{1 / p}\right)$.

In general, for $n>1$, we have by Lemma 2.7 , 
(11)

$$
\begin{aligned}
\phi_{n} & =\phi_{n-1}^{p}-p h_{n-1}+\left[\left[p^{2}\right]\right] \\
& =\phi_{n-1}^{p}-p\left(h_{n-2}^{p}+[[p]]\right)+\left[\left[p^{2}\right]\right] \\
& =\phi_{n-1}^{p}-p h_{n-2}^{p}+\left[\left[p^{2}\right]\right] \\
& =\phi_{n-1}^{p}-\left(p^{1 / p} h_{n-2}\right)^{p}+\left[\left[p^{2}\right]\right] \\
& =\left(\phi_{n-1}-p^{1 / p} h_{n-2}\right)^{p}-p g_{0}\left(\phi_{n-1}, p^{1 / p} h_{n-2}\right)+\left[\left[p^{2}\right]\right] \\
& =\left(\phi_{n-1}-p^{1 / p} h_{n-2}\right)^{p}-p^{\alpha_{2}} \phi_{n-1}^{p-1} h_{n-2}+\left[\left[p^{\alpha_{2}+1 / p}\right]\right] .
\end{aligned}
$$

(For the last equality, note that

$$
\begin{aligned}
p g_{0}\left(\phi_{n-1}, p^{1 / p} h_{n-2}\right)= & p\left(p^{1 / p}\right) h_{n-2} \phi_{n-1}^{p-1} \\
& +\left(\begin{array}{c}
p \\
2
\end{array}\right)\left(p^{1 / p} h_{n-2}\right)^{2} \phi_{n-1}^{p-2}+\cdots .
\end{aligned}
$$

Also, note that $p^{1+1 / p}=p^{\alpha_{2}}$, and $p^{1+2 / p}=p^{\alpha_{2}+1 / p}$. Finally, note that since $p \geq 3,1+2 / p<2$.)

Claim. For $2 \leq k \leq n-1$, if

$$
S_{k}=a_{k}^{p}-p^{\alpha_{k}} \phi_{n-1}^{p-1} \phi_{n-2}^{p-1} \cdots \phi_{n-k+1}^{p-1} h_{n-k}+\left[\left[p^{\alpha_{2}+1 / p}\right]\right],
$$

for some $a_{k} \in F$ with $a_{k}=\phi_{n-1}+\left[\left[p^{1 / p}\right]\right]$, then

$$
S_{k}=a_{k+1}^{p}-p^{\alpha_{k+1}} \phi_{n-1}^{p-1} \phi_{n-2}^{p-2} \cdots \phi_{n-k}^{p-1} h_{n-k-1}+\left[\left[p^{\alpha_{2}+1 / p}\right]\right],
$$

for some $a_{k+1} \in F$ with $a_{k+1}=\phi_{n-1}+\left[\left[p^{1 / p}\right]\right]$.

Proof of claim. For, by Lemma 2.7, $\phi_{j}=\phi_{j-1}^{p}+[[p]]$ and $h_{j}=$ $h_{j-1}^{p}+[[p]]$ for all $j \geq 1$, so

$$
\begin{aligned}
S_{k}= & a_{k}^{p}-p^{\alpha_{k}}\left(\phi_{n-2}^{p}+[[p]]\right)^{p-1}\left(\phi_{n-3}^{p}+[[p]]\right)^{p-1} \\
& \cdots\left(\phi_{n-k}^{p}+[[p]]\right)^{p-1}\left(h_{n-k-1}^{p}+[[p]]\right)+\left[\left[p^{\alpha_{2}+1 / p}\right]\right] \\
= & a_{k}^{p}-p^{\alpha_{k}}\left(\phi_{n-2}^{p(p-1)}+[[p]]\right)\left(\phi_{n-3}^{p(p-1)}+[[p]]\right) \\
& \left.\cdots\left(\phi_{n-k}^{p(p-1)}+[[p]]\right)\left(h_{n-k-1}^{p}+[[p]]\right)+\left[\left[p^{\alpha_{2}+1 / p}\right]\right]\right] \\
= & a_{k}^{p}-p^{\alpha_{k}}\left(\phi_{n-2}\right)^{p(p-1)} \cdots\left(\phi_{n-k}\right)^{p(p-1)} h_{n-k-1}^{p} \\
& \left.+\left[\left[p^{\alpha_{k}+1}\right]\right]+\left[\left[p^{\alpha_{2}+1 / p}\right]\right]\right] \\
= & a_{k}^{p}-\left(p^{\alpha_{k} / p}\left(\phi_{n-2}\right)^{(p-1)} \cdots\left(\phi_{n-k}\right)^{(p-1)} h_{n-k-1}\right)^{p}+\left[\left[p^{\alpha_{2}+1 / p}\right]\right] \\
= & \left(a_{k}-p^{\alpha_{k} / p} \phi_{n-2}^{p-1} \cdots \phi_{n-k}^{p-1} h_{n-k-1}\right)^{p} \quad\left(\text { as } \alpha_{2}+1 / p<\alpha_{k}+1\right) \\
& -p g_{0}\left(a_{k}, p^{\alpha_{k} / p} \phi_{n-2}^{p-1} \cdots \phi_{n-k}^{p-1} h_{n-k-1}\right)+\left[\left[p^{\alpha_{2}+1 / p}\right]\right] .
\end{aligned}
$$


Expanding $p g_{0}\left(a_{k}, p^{\alpha} / p \phi_{n-2}^{p-1} \cdots \phi_{n-k}^{p-1} h_{n-k-1}\right)$ and considering the first two terms of lowest value, we find

$$
\begin{aligned}
S_{k}= & \left(a_{k}-p^{\alpha_{k} / p} \phi_{n-2}^{p-1} \cdots \phi_{n-k}^{p-1} h_{n-k-1}\right)^{p} \\
& -p^{1+\alpha_{k} / p} a_{k}^{p-1} \phi_{n-2}^{p-1} \cdots \phi_{n-k}^{p-1} h_{n-k-1}+\left[\left[p^{1+\left(2 \alpha_{k} / p\right)}\right]\right]+\left[\left[p^{\alpha_{2}+1 / p}\right]\right] .
\end{aligned}
$$

Now $1+\alpha_{k} / p=\alpha_{k+1}$. Also, $1+\left(2 \alpha_{k} / p\right)=\alpha_{k+1}+\alpha_{k} / p>\alpha_{2}+1 / p$ (as $\alpha_{k+1}>\alpha_{2}$ and $\alpha_{k}>1$ when $k \geq 2$ ). Thus,

$$
\begin{aligned}
S_{k}= & \left(a_{k}-p^{\alpha_{k} / p} \phi_{N-2}^{p-1} \cdots \phi_{n-k}^{p-1} h_{n-k-1}\right)^{p} \\
& -p^{\alpha_{k+1}} a_{k}^{p-1} \phi_{n-2}^{p-1} \cdots \phi_{n-k}^{p-1} h_{n-k-1}+\left[\left[p^{\alpha_{2}+1 / p}\right]\right] .
\end{aligned}
$$

Now recalling that $a_{k}=\phi_{n-1}+\left[\left[p^{1 / p}\right]\right]$, we find $a_{k}^{p-1}=\phi_{n-1}^{p-1}+\left[\left[p^{1 / p}\right]\right]$. Hence,

$$
\begin{aligned}
S_{k}= & \left(a_{k}-p^{\alpha_{k} / p} \phi_{n-2}^{p-1} \cdots \phi_{n-k}^{p-1} h_{n-k-1}\right)^{p} \\
& -p^{\alpha_{k+1}} \phi_{n-1}^{p-1} \phi_{n-2}^{p-1} \cdots \phi_{n-k}^{p-1} h_{n-k-1}+\left[\left[p^{\alpha_{k+1}+1 / p}\right]\right]+\left[\left[p^{\alpha_{2}+1 / p}\right]\right] .
\end{aligned}
$$

Since $\alpha_{k+1}>\alpha_{2}, \alpha_{k+1}+1 / p>\alpha_{2}+1 / p$. Thus,

$$
\begin{aligned}
S_{k}= & \left(a_{k}-p^{\alpha_{k} / p} \phi_{n-2}^{p-1} \cdots \phi_{n-k}^{p-1} h_{n-k-1}\right)^{p} \\
& -p^{\alpha_{k+1}} \phi_{n-1}^{p-1} \phi_{n-2}^{p-1} \cdots \phi_{n-k}^{p-1} h_{n-k-1}+\left[\left[p^{\alpha_{2}+1 / p}\right]\right] .
\end{aligned}
$$

Take $a_{k+1}=\left(a_{k}-p^{\alpha_{k} / p} \phi_{n-2}^{p-1} \cdots \phi_{n-k}^{p-1} h_{n-k-1}\right)$. Since $a_{k}=\phi_{n-1}+$ $\left[\left[p^{1 / p}\right]\right]$ and since $1 / p<\alpha_{k} / p($ as $k \geq 2), a_{k+1}=\phi_{n-1}+\left[\left[p^{1 / p}\right]\right]$. This proves the claim.

Proof of Case 1 (continued). We now use the claim above to inductively reduce (11) until it yields

$$
\phi_{n}=a^{p}+p^{\alpha_{n}} b h_{0}+\delta,
$$

for some $a \in F$ with $v(a)=0$, some $b \in F$ with $v(b)=0$ and $\bar{b} \in \bar{F}^{p}$, and some $\delta \in F$ with $v(\delta)>\alpha_{n}$. Since $p^{\alpha_{n} / p}=$ $p^{1 / p+1 / p^{2}+\cdots+1 / p^{n}} \in F_{0}$, it will follow immediately from Lemma 2.2 that $v$ extends uniquely from $F$ to $H_{n}$, and $\overline{H_{n}}=\bar{F}\left(h_{0}^{1 / p}\right)$.

If $n=2$, then $(11)$ is already in the desired form, since $\overline{\phi_{1}} \in \bar{F}^{p}$. Otherwise, we write (11) as

$$
\phi_{n}=S_{2}+\left[\left[p^{\alpha_{2}+1 / p}\right]\right],
$$


with $a_{2}=\phi_{n-1}-p^{1 / p} h_{n-2}$. By repeatedly applying the claim, we find

$$
\phi_{n}=S_{n}+\left[\left[p^{\alpha_{2}+1 / p}\right]\right],
$$

with $S_{n}=a_{n}^{p}-p^{\alpha_{n}} \phi_{n-1}^{p-1} \cdots \phi_{1}^{p-1} h_{0}$, for some $a_{n} \in F$ with $a_{n}=\phi_{n-1}+$ $\left[\left[p^{1 / p}\right]\right]$. By Lemma 2.8, $\alpha_{n}<\alpha_{2}+1 / p$ for all $n \geq 3$. Observing that the residues of $\phi_{n-1}, \ldots, \phi_{1}$ are all $p$ th powers in $\bar{F}$, we find that $\phi_{n}$ is now in the form (12), and we are done.

Case $2(p=2)$. The basic steps for the $p=2$ case are the same as for the odd $p$ case, the differences are only in the details.

If $n=1$, then, by Lemma $2.7, \phi_{1}=\psi_{0}^{2}-2\left(h_{0}+e_{1}\right)+[[4]]$, so by Lemma 2.2, $v$ extends uniquely to $H_{1}$, and $\overline{H_{1}}=\bar{F}\left(\sqrt{\left(h_{0}+e_{1}\right)}\right)$. But $e_{1}$ is already a square in $\bar{F}$, so $\overline{H_{1}}=\bar{F}\left(\sqrt{h_{0}}\right)$.

In general, for $n>1$, we have, by Lemma 2.7

$$
\begin{aligned}
\phi_{n}= & \psi_{n-1}^{2}-2\left(h_{n-1}+e_{n}\right)+[[4]] \\
= & \psi_{n-1}^{2}-2\left(h_{n-2}^{2}+[[2]]+e_{n-1}^{2}+[[2]]\right)+[[4]] \\
= & \psi_{n-1}^{2}-2\left(h_{n-2}^{2}+e_{n-1}^{2}\right)+[[4]] \\
= & \psi_{n-1}^{2}-2\left(\left(h_{n-2}+e_{n-1}\right)^{2}+[[2]]\right)+[[4]] \\
= & \psi_{n-1}^{2}-2\left(h_{n-2}+e_{n-1}\right)^{2}+[[4]] \\
= & \psi_{n-1}^{2}+2\left(h_{n-2}+e_{n-1}\right)^{2}-4\left(h_{n-2}+e_{n-1}\right)^{2}+[[4]] \\
= & \psi_{n-1}^{2}+\left(2^{1 / 2}\left(h_{n-2}+e_{n-1}\right)\right)^{2}+[[4]] \\
= & \left(\psi_{n-1}+\left(2^{1 / 2}\left(h_{n-2}+e_{n-1}\right)\right)\right)^{2} \\
& -2(2)^{1 / 2} \psi_{n-1}\left(h_{n-2}+e_{n-1}\right)+[[4]] \\
= & \left(\psi_{n-1}+\left(2^{1 / 2}\left(h_{n-2}+e_{n-1}\right)\right)\right)^{2} \\
& -2^{\alpha_{2}} \psi_{n-1}\left(h_{n-2}+e_{n-1}\right)+[[4]] .
\end{aligned}
$$

Claim. For $2 \leq k \leq n-1$, let

$$
S_{k}=a_{k}^{2}-2^{\alpha_{k}} \psi_{n-1} \cdots \psi_{n-k+1}\left(h_{n-k}+e_{n-k+1}\right)+[[4]],
$$

for some $a_{k} \in F$ with $a_{k}=\psi_{n-1}+\left[\left[2^{1 / 2}\right]\right]$. Then,

$$
S_{k}=a_{k+1}^{2}-2^{\alpha_{k}+1} \psi_{n-1} \cdots \psi_{n-k}\left(h_{n-k-1}+e_{n-k}\right)+[[4]],
$$

for some $a_{k+1} \in F$ with $a_{k+1}=\psi_{n-1}+\left[\left[2^{1 / 2}\right]\right]$. 
Proof of Claim. We have

$$
\begin{aligned}
S_{k}= & a_{k}^{2}-\left(2^{\alpha_{k} / 2}\right)^{2}\left(\psi_{n-2}^{2}+[[2]]\right) \\
& \cdots\left(\psi_{n-k}^{2}+[[2]]\right)\left(h_{n-k-1}^{2}+[[2]]+e_{n-k}^{2}+[[2]]\right)+[[4]] \\
= & a_{k}^{2}-\left(2^{\alpha_{k} / 2}\right)^{2} \psi_{n-2}^{2} \cdots \psi_{n-k}^{2}\left(h_{n-k-1}^{2}+e_{n-k}^{2}\right) \\
& +\left[\left[2^{1+2\left(\alpha_{k} / 2\right)}\right]\right]+[[4]] \\
= & a_{k}^{2}-\left(2^{\alpha_{k} / 2}\right)^{2} \psi_{n-2}^{2} \cdots \psi_{n-k}^{2}\left(\left(h_{n-k-1}+e_{n-k}\right)^{2}+[[2]]\right)+[[4]] \\
= & a_{k}^{2}-\left(2^{\alpha_{k} / 2} \psi_{n-2} \cdots \psi_{n-k}\left(h_{n-k-1}+e_{n-k}\right)\right)^{2}+[[4]] \\
= & a_{k}^{2}+\left(2^{\alpha_{k} / 2} \psi_{n-2} \cdots \psi_{n-k}\left(h_{n-k-1}+e_{n-k}\right)\right)^{2} \\
& -2\left(2^{\alpha_{k} / 2} \psi_{n-2} \cdots \psi_{n-k}\left(h_{n-k-1}+e_{n-k}\right)\right)^{2}+[[4]] \\
= & a_{k}^{2}+\left(2^{\alpha_{k} / 2} \psi_{n-2} \cdots \psi_{n-k}\left(h_{n-k-1}+e_{n-k}\right)\right)^{2}+[[4]] \\
= & \left(a_{k}+2^{\alpha_{k} / 2} \psi_{n-2} \cdots \psi_{n-k}\left(h_{n-k-1}+e_{n-k}\right)\right)^{2} \\
& -2\left(2^{\alpha_{k} / 2}\right) a_{k} \psi_{n-2} \cdots \psi_{n-k}\left(h_{n-k-1}+e_{n-k}\right)+[[4]] \\
= & \left(a_{k}+2^{\alpha_{k} / 2} \psi_{n-2} \cdots \psi_{n-k}\left(h_{n-k-1}+e_{n-k}\right)\right)^{2} \\
& -2^{1+\alpha k / 2}\left(\psi_{n-1}+\left[\left[2^{1 / 2}\right]\right]\right) \psi_{n-2} \\
& \cdots \psi_{n-k}\left(h_{n-k-1}+e_{n-k}\right)+[[4]] \\
= & \left(a_{k}+2^{\alpha_{k} / 2} \psi_{n-2} \cdots \psi_{n-k}\left(h_{n-k-1}+e_{n-k}\right)\right)^{2} \\
& -2^{\alpha_{k+1}} \psi_{n-1} \psi_{n-2} \cdots \psi_{n-k}\left(h_{n-k-1}+e_{n-k}\right) \\
& +\left[\left[2^{\alpha_{k+1}+1 / 2}\right]\right]+[[4]] \\
= & a_{k+1}^{2}-2^{\alpha_{k+1}} \psi_{n-1} \psi_{n-2} \cdots \psi_{n-k}\left(h_{n-k-1}+e_{n-k}\right)+[[4]],
\end{aligned}
$$

where

$$
a_{k+1}=a_{k}+2^{\alpha_{k} / 2} \psi_{n-2} \cdots \psi_{n-k}\left(h_{n-k-1}+e_{n-k}\right),
$$

(so $\left.a_{k+1}=\psi_{n-1}+\left[\left[2^{1 / 2}\right]\right]+\left[\left[2^{\alpha_{k} / 2}\right]\right]=\psi_{n-1}+\left[\left[2^{1 / 2}\right]\right]\right)$.

Proof of Case 2 (continued). We now use the claim above to inductively reduce (13) until it yields

$$
\phi_{n}=a^{2}+2^{\alpha_{n}} b\left(h_{0}+e_{1}\right)+\delta,
$$

for some $a \in F$ with $v(a)=0$, some $b \in F$ with $v(b)=0$ and $\bar{b} \in \bar{F}^{2}$, and some $\delta \in F$ with $v(\delta)>\alpha_{n}$. Since $2^{\alpha_{n} / 2}=$ $2^{1 / 2+1 / 2^{2}+\cdots+1 / 2^{n}} \in F_{0}$, it will follow immediately from Lemma 2.2 that $v$ extends uniquely from $F$ to $H_{n}$, and $\overline{H_{n}}=\bar{F}\left(\sqrt{h_{0}+e_{1}}\right)=$ $\bar{F}\left(\sqrt{h_{0}}\right)$.

If $n=2$, then (13) is already in the desired form, since $\overline{\psi_{1}} \in \bar{F}^{2}$. 
Otherwise, we write (13) as

$$
\phi_{n}=S_{2}+[[4]],
$$

with $a_{2}=\psi_{n-1}+2^{1 / 2}\left(h_{n-2}+e_{n-1}\right)$. By repeatedly applying the claim, we find

$$
\phi_{n}=S_{n}+[[4]] \text {, }
$$

with $S_{n}=a_{n}^{2}-\left(2^{\alpha_{n}}\right) \psi_{n-1} \cdots \psi_{1}\left(h_{0}+e_{1}\right)$, for some $a_{n} \in F$ with $a_{n}=$ $\psi_{n-1}+\left[\left[2^{1 / 2}\right]\right]$. By Lemma 2.8 (or by more direct means), $\alpha_{n}<2$ for all $n \geq 3$. Observing that the residues of $\psi_{n-1}, \ldots, \psi_{1}$ are all squares in $\bar{F}$, we find that $\phi_{n}$ is now in the form (15), and we are done.

3. The Dec results. Let $F_{0}$ be a field of characteristic 0 containing all primitive $p^{i}$ th roots of unity $\omega_{i}(i=1,2, \ldots)$, chosen so that $\omega_{i+1}^{p}=\omega_{i}$. (We will write $\omega$ for $\omega_{1}$.) If $L \supseteq F_{0}$ is any field, and if $a$ and $b$ are in $L^{*}$, then, as in [D, Chapter 11$],\left(a, b ; p^{n}, L, \omega_{n}\right)$ will denote the algebra generated over $L$ by two symbols $\alpha$ and $\beta$ subject to $\alpha^{p^{n}}=a, \beta^{p^{n}}=b$, and $\alpha \beta=\omega_{n} \alpha \beta$, and will be referred to as a symbol algebra. Now let $F=F_{0}\left(x_{1}, x_{2}, y\right)$ be the rational function field over $F_{0}$ in the three indeterminates $x_{1}, x_{2}$, and $y$. For each $n \geq 1$, define

$$
A_{n}=\left(x_{1}, x_{1}^{p^{n}}-y ; p, F, \omega\right) \otimes_{F}\left(x_{2}, x_{2}^{p^{n}}-y ; p, F, \omega\right) .
$$

Lemma 3.1. For each $n \geq 1, A_{n}$ has index $p^{2}$ and exponent $p$. Further,

$$
A_{n} \sim\left(y, \frac{\left(x_{1}^{p^{n}}-y\right)\left(x_{2}^{p^{n}}-y\right)}{x_{1}^{p^{n}} x_{2}^{p^{n}}} ; p^{n+1}, F, \omega_{n+1}\right) .
$$

Proof. This is very similar to the proof of Proposition 2 in [Se2], and we only sketch the proof. The factor $\left(x_{1}, x_{1}^{p^{n}}-y ; p, F, \omega\right)$ is NSR with respect to the $x_{1}$-adic valuation on $F$, with residue isomorphic to $F_{0}\left(x_{2}, z\right)$, where $z=y^{1 / p}$. The factor $\left(x_{2}, x_{2}^{p^{n}}-y ; p, F_{0}\left(x_{2}, z\right), \omega\right)$ (i.e., defined over $F_{0}\left(x_{2}, z\right)$ ) is NSR with respect to the $x_{2}^{p^{n-1}}-z$ adic valuation (with residue isomorphic to $F_{0}\left(x_{2}^{1 / p}\right)$ ). It follows from [ $\mathbf{J W}$, Theorem 5.15] that $A_{n}$ has index $p^{2}$. It is clear that $\exp \left(A_{n}\right)=p$. As for the final statement of the lemma, standard symbol algebra identities (e.g., [D, Chapter 11, pages 77-82]) along with the assumption 
about the roots of unity in $F_{0}$ show that

$$
\begin{aligned}
A_{n} \sim & \left(x_{1}^{p^{n}}, x_{1}^{p^{n}}-y ; p^{n+1}, F, \omega_{n+1}\right) \\
& \otimes_{F}\left(x_{2}^{p^{n}}, x_{2}^{p^{n}}-y ; p^{n+1}, F, \omega_{n+1}\right) \\
\sim & \left(-y, \frac{x_{1}^{p^{n}}-y}{x_{1}^{p^{n}}} ; p^{n+1}, F, \omega_{n+1}\right) \\
& \otimes_{F}\left(-y, \frac{x_{2}^{p^{n}}-y}{x_{2}^{p^{n}}} ; p^{n+1}, F, \omega_{n+1}\right) \\
\sim & \left(y, \frac{\left(x_{1}^{p^{n}}-y\right)\left(x_{2}^{p^{n}}-y\right)}{x_{1}^{p^{n}} x_{2}^{p^{n}}} ; p^{n+1}, F, \omega_{n+1}\right) .
\end{aligned}
$$

Now write $\phi_{n}$ for $\left(x_{1}^{p^{n}}-y\right)\left(x_{2}^{p^{n}}-y\right)$ (this notation will be seen to be consistent with that of $\S 2)$, and write $K_{n}$ for the field $F\left(y^{1 / p^{n}}, \phi_{n}^{1 / p}\right)$. Then $A_{n} \in \operatorname{Br}\left(K_{n} / F\right)$. Tignol ([T2, Theorem 1]) showed that when $p$ is odd, $A_{1} \notin \operatorname{Dec}\left(K_{1} / F\right)$. We have

THEOREM 3.2. 1. For $p$ odd and $n \geq 1$, or $p=2$ and $n \geq 2$, $A_{n} \notin \operatorname{Dec}\left(K_{n} / F\right)$.

2. More generally, for $p$ odd, $n \geq 1$, and $0 \leq l \leq n-1$, or $p=2$, $n \geq 2$, and $0 \leq l \leq n-2$, let $F_{l}=F\left(y^{1 / p^{l}}\right)$ (so $\left.F_{l} \subset K_{n}\right)$. Then, $A_{n} \otimes_{F} F_{l} \notin \operatorname{Dec}\left(K_{n} / F_{l}\right)$.

3. Further, let $E$ be any finite extension of $F$, with $p \nmid[E: F]$. For $p$ odd, $n \geq 1$, and $0 \leq l \leq n-1$, or $p=2, n \geq 2$, and $0 \leq l \leq n-2$, let $E_{l}=E\left(y^{1 / p^{l}}\right)\left(\right.$ so $\left.E_{l} \subset K_{n} \cdot E\right)$. Then, $A_{n} \otimes_{F} E_{l} \notin \operatorname{Dec}\left(K_{n} \cdot E / E_{l}\right)$.

Proof of Theorem 3.2. It is clearly sufficient to prove (3). Moreover, it is sufficient to prove (3) for the case $l=n-1$ (for $p$ odd) and $l=n-2$ (for $p=2$ ). For, assume that for $l<n-1$ and $p$ odd, or for $l<n-2$ and $p=2$,

$$
A_{n} \otimes_{F} E_{l} \sim\left(y^{1 / p^{l}}, b_{1} ; p^{n-l}, E_{l}, \omega_{n-l}\right) \otimes_{E_{l}}\left(b_{2}, \phi_{n} ; p, E_{l}, \omega\right),
$$

for some $b_{1}$ and $b_{2} \in E_{l}^{*}$. Then, extending scalars to $E_{n-1}$ (for $p$ odd) and $E_{n-2}$ (for $p=2$ ), we find by standard symbol algebra identities

$$
A_{n} \otimes_{F} E_{n-1} \sim\left(y^{1 / p^{n-1}}, b_{1} ; p, E_{n-1}, \omega\right) \otimes_{E_{n-1}}\left(b_{2}, \phi_{n} ; p, E_{n-1}, \omega\right)
$$

for $p$ odd, and

$A_{n} \otimes_{F} E_{n-2} \sim\left(y^{1 / p^{n-2}}, b_{1} ; p^{2}, E_{n-2}, \omega_{2}\right) \otimes_{E_{n-2}}\left(b_{2}, \phi_{n} ; p, E_{n-2}, \omega\right)$ 
for $p=2$. Thus, we find that for $p$ odd and $l<n-1$, if

$$
A_{n} \otimes_{F} E_{l} \in \operatorname{Dec}\left(K_{n} \cdot E / E_{l}\right)
$$

then

$$
A_{n} \otimes_{F} E_{n-1} \in \operatorname{Dec}\left(K_{n} \cdot E / E_{n-1}\right),
$$

and for $p=2$ and $l<n-2$, if

$$
A_{n} \otimes_{F} E_{l} \in \operatorname{Dec}\left(K_{n} \cdot E / E_{l}\right)
$$

then

$$
A_{n} \otimes_{F} E_{n-2} \in \operatorname{Dec}\left(K_{n} \cdot E / E_{n-2}\right) .
$$

We find it convenient at this point to divide the proof according to whether $p$ is odd or even.

Case 1 ( $p$ odd). Assume that $A_{n} \otimes_{F} E_{n-1} \sim\left(y^{1 / p^{n-1}}, b_{1} ; p, E_{n-1}, \omega\right) \otimes E_{n-1}\left(b_{2}, \phi_{n} ; p, E_{n-1}, \omega\right)$, for some $b_{1}$ and $b_{2} \in E_{n-1}^{*}$. By Lemma 3.1 and standard symbol algebra identities,

$$
A_{n} \otimes_{F} E_{n-1} \sim\left(y^{1 / p^{n-1}}, \frac{\phi_{n}}{x_{1}^{p^{n}} x_{2}^{p^{n}}} ; p^{2}, E_{n-1}, \omega_{2}\right) .
$$

Put $z=y^{1 / p^{n}}$. Then, extending scalars further to $E_{n}=E(z)$, and noting that $x_{1}^{p^{n}}$ and $x_{2}^{p^{n}}$ are $p$ th powers, we find

$$
\left(z, \phi_{n} ; p, E_{n}, \omega\right) \sim\left(b, \phi_{n} ; p, E_{n}, \omega\right),
$$

where we have written $b$ for $b_{2}$. Hence,

$$
\left(z / b, \phi_{n} ; p, E_{n}, \omega\right) \sim 1,
$$

so

$$
z / b=N(u)
$$

for some $u \in E_{n}\left(\left(\phi_{n}\right)^{1 / p}\right)$, where $N$ denotes the norm from $E_{n}\left(\left(\phi_{n}\right)^{1 / p}\right)$ to $E_{n}$. We will prove that it is impossible to find $b \in E_{n-1}$ and $u \in E_{n}\left(\left(\phi_{n}\right)^{1 / p}\right)$ such that (16) holds.

If $\overline{F_{0}}$ denotes the algebraic closure of $F_{0}$, then $\overline{F_{0}}\left(x_{1}, x_{2}, y\right)$ is normal over $F_{0}\left(x_{1}, x_{2}, y\right)$, so if $E=F_{0}\left(x_{1}, x_{2}, y\right)(t)$ for some $t \in$ $E^{*}$, then it is standard that the degree of the minimum polynomial of $t$ over $\overline{F_{0}}\left(x_{1}, x_{2}, y\right)$ divides the degree of the minimum polynomial of $t$ over $F_{0}\left(x_{1}, x_{2}, y\right)$. Hence $p \nmid\left[E \cdot \overline{F_{0}}\left(x_{1}, x_{2}, y\right): \overline{F_{0}}\left(x_{1}, x_{2}, y\right)\right]$. Thus, while showing that (15) cannot hold, we may assume that $F_{0}$ is 
algebraically closed. In particular, we may assume that $F_{0}$ contains $p^{1 / p^{i}}$ for all $i(i=1,2, \ldots)$, so we may apply the machinery of $\S 2$.

Now write $\chi$ for $h_{0}\left(x_{1}, x_{2}, z\right)$, where $h_{0}$ is as in $\S 2$. As with the polynomial $h_{0}$, we will abuse notation and continue to write $\chi$ for the residue of $h_{0}$ under appropriate $p$-adic valuations. Observe that over $E_{n}, \phi_{n}=\left(x_{1}^{p^{n}}-z^{p^{n}}\right)\left(x_{2}^{p^{n}}-z^{p^{n}}\right)$, which, after renaming variables is indeed the same as the " $\phi_{n}$ " of $\S 2$.

We first need an easy lemma:

LEMMA 3.3. Let $p$ be a prime, and let $(F, v)$ be a valued field. Let $K$ be a finite dimensional separable extension of $F$ such that $p \nmid[K: F]$. Then for some extension of $v$ to $K, p \nmid[\bar{K}: \bar{F}]$.

Proof. Let $v_{i}(1 \leq i \leq s)$ be the extensions of $v$ to $K$, and let $(\bar{K})_{i}$ denote the residues of $K$ with respect to the valuations $v_{i}$. Let $F_{h}$ denote the henselization of $F$ with respect to $v$, and let $K_{i, h}$ denote the henselization of $K$ with respect to $v_{i}(1 \leq i \leq s)$. Then (by [E, Theorem 17.17]) $[K: F]=\sum_{i=1}^{s}\left[K_{i, h}: F_{h}\right]$, so if $p \nmid[K: F]$, then $p \nmid\left[K_{i, h}: F_{h}\right]$ for some $i$. Now $\overline{K_{i, h}}=(\bar{K})_{i}$ and $\overline{F_{h}}=\bar{F}$, so by Ostrowski's theorem ([O, Satz 4], see also [E, Theorem 20.21]), $\left[(\bar{K})_{i}: \bar{F}\right] \mid\left[K_{i, h}: F_{h}\right]$. Hence, for this $i, p \nmid\left[(\bar{K})_{i}: \bar{F}\right]$.

Proof of Theorem 3.2 (continued). Now let $L=F_{0}\left(x_{1}, x_{2}, z\right)$ and let $v$ be the standard extension of any $p$-adic valuation on $F_{0}$ to $L$ (so $\bar{L}=\overline{F_{0}}\left(x_{1}, x_{2}, z\right)$ ). Let $L_{1}=F_{0}\left(x_{1}, x_{2}, z^{p}\right)$, and let $v_{L_{1}}$ denote the restriction of $v$ to $L_{1}$. Choose an extension $w$ of $v_{L_{1}}$ to $E_{n-1}$ such that $p \nmid\left[\overline{E_{n-1}}: \overline{L_{1}}\right]$. (Since $\left[E_{n-1}: L_{1}\right]=[E: F]$, the lemma above shows that such a choice is possible.) By Proposition $2.4 v$ extends uniquely from $L$ to $L\left(\phi_{n}^{1 / p}\right)$, with residue $\bar{L}\left(\chi^{1 / p}\right)$. Since

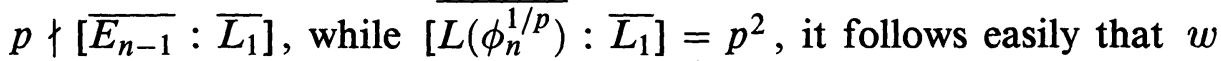
extends uniquely from $E_{n-1}$ to $E_{n}\left(\phi_{n}^{1 / p}\right)$, with residue $\overline{E_{n}}\left(\chi^{1 / p}\right)$.

Now, continue to write $w$ for the (unique) extension of $w$ to $E_{n}\left(\phi_{n}^{1 / p}\right)$ and consider the relation (15). Since $v(z)=0$, we get $w(b)+w(N(u))=0$. Since $\Gamma_{E_{n-1}}=\Gamma_{E_{n}\left(\phi_{n}^{1 / p}\right)}$, there is a $c \in E_{n-1}$ such that $w(c)=w(u)$. Then, $b N(u)=b c^{p} N(u / c)$, and $w(u / c)=0$, $w\left(b c^{p}\right)=w(b)+p \cdot w(u)=w(b)+w(N(u))=0$, and of course, $b c^{p} \in E_{n-1}$. Hence, we may assume in (15) that $w(b)=w(u)=0$.

Now let $\sigma$ be a generator of $\mathscr{G}\left(E_{n}\left(\phi_{n}^{1 / p}\right) / E_{n}\right)$, so

$$
N(u)=u \cdot \sigma(u) \cdots \sigma^{p-1}(u) .
$$


Hence, $\overline{N(u)}=\bar{u} \cdot \bar{\sigma}(\bar{u}) \cdots \bar{\sigma}^{p-1}(\bar{u})$, where $\bar{\sigma}$ is the induced automorphism of $\overline{E_{n}}\left(\chi^{1 / p}\right) / \overline{E_{n}}$ (i.e., $\bar{\sigma}(\bar{x})=\overline{\sigma(x)}$ for all $\bar{x} \in \overline{E_{n}}\left(\chi^{1 / p}\right)$ ). Since the extension $\overline{E_{n}}\left(\chi^{1 / p}\right) / \overline{E_{n}}$ is purely inseparable, $\bar{\sigma}$ is just the identity, so find $\overline{N(u)}=\bar{u}^{p}$. Thus, reducing the relation $z=b N(u) \bmod -$ ulo the maximal ideal of the valuation ring of $w$, we find $z=\bar{b} \bar{u}^{p}$, where $\bar{b} \in \overline{E_{n-1}}$, and $\bar{u} \in \overline{E_{n}}\left(\chi^{1 / p}\right)$. We will show that such a relation is impossible.

Let $\overline{E_{n-1}}=\overline{L_{1}}(\theta)$, so that $1, \theta, \ldots, \theta^{s-1}$ form a basis for $\overline{E_{n-1}} / \overline{L_{1}}$, with $s=\left[\overline{E_{n-1}}: \overline{L_{1}}\right]$. Since $p \nmid s$, it follows easily that $\overline{E_{n-1}}=$ $\overline{L_{1}}\left(\theta^{p}\right)$, and $1, \theta^{p}, \ldots, \theta^{(s-1) p}$ also form a basis of $\overline{E_{n_{1}}} / \overline{L_{1}}$. Likewise, $1, \theta, \ldots, \theta^{s-1}$, as well as $1, \theta^{p}, \ldots, \theta^{(s-1) p}$, are both bases of $\overline{E_{n}}\left(\chi^{1 / p}\right) / \bar{L}\left(\chi^{1 / p}\right)$. Now let

$$
1 / \bar{b}=b_{0}+b_{1} \theta^{p}+\cdots+b_{s-1} \theta^{(s-1) p},
$$

where the $b_{i} \in \overline{L_{1}}(i=0,1, \ldots, s-1)$. Similarly, let

$$
\bar{u}=u_{0}+u_{1} \theta+\cdots+u_{s-1} \theta^{s-1},
$$

where the $u_{i} \in \bar{L}\left(\chi^{1 / p}\right)(i=0,1, \ldots, s-1)$. Substituting the expressions above for $1 / \bar{b}$ and $\bar{u}$ in $z / \bar{b}=\bar{u}^{p}$ and comparing like terms, we find

$$
z b_{0}=u_{0}^{p},
$$

where of course, $b_{0} \in \overline{L_{1}}$ and $u_{0} \in \bar{L}\left(\chi^{1 / p}\right)$. The impossibility of (16) above is just the impossibility of [T2, (23)], and follows immediately from the proof given there. However, for the sake of completeness, we will reprove this result here. Our proof will be different from that in [T2]; instead, it will be similar in spirit to the proof below of a corresponding result for $p=2$.

Write $c$ for $1 / b_{0}$ and $u$ for $u_{0}$, so we need to show that there do not exist $c \in \overline{L_{1}}\left(=\overline{F_{0}}\left(x_{1}, x_{2}, z^{p}\right)\right)$ and $u \in \bar{L}\left(\chi^{1 / p}\right)$ $\left(=\overline{F_{0}}\left(x_{1}, x_{2}, z\right)\left(\chi^{1 / p}\right)\right)$ such that $z / c=u^{p}$. By considering the $z$ adic valuation on $\overline{L_{1}}$, it is easy to see that for any $c \in \bar{L}_{1}{ }^{*} z / c \notin \bar{L}^{* p}$. Now assume that $z / c=u^{p}$ for some $c \in \bar{L}_{1}{ }^{*}$ and some $u \in \bar{L}\left(\chi^{1 / p}\right)$. Then $\bar{L}\left((z / c)^{1 / p}\right) \subset \bar{L}\left(\chi^{1 / p}\right)$, so we find $\bar{L}\left((z / c)^{1 / p}\right)=\bar{L}\left(\chi^{1 / p}\right)$. Thus, there exist $f_{i} \in \bar{L}^{p}(i=0,1, \ldots, p-1)$ such that

$$
\chi\left(=h_{0}\left(x_{1}, x_{2}, z\right)\right)=\sum_{i=1}^{p-1} f_{i}(z / c)^{i}
$$


Since $1, z, \ldots, z^{p-1}$ form a basis for $L / \overline{L_{1}}$, we may write

$$
h_{0}\left(x_{1}, x_{2}, z\right)=\sum_{i=0}^{p-1} e_{i} z^{i} \quad \text { for } e_{i} \in \overline{L_{1}},
$$

where the values of the $e_{i}$ may be derived from the definition of $h_{0}$ in (6). Then, (17) takes the form

$$
c^{p-1}\left(\sum_{i=0}^{p-1} e_{i} z^{i}\right)=\sum_{i=0}^{p-1} c^{p-1-i} f_{i} z^{i}
$$

Now $c \in \overline{L_{1}}$, and $\bar{L}^{p} \subset \overline{L_{1}}$. Hence, comparing the coefficients of $z^{i}$ in (18), we find $c^{i} e_{i}=f_{i}(i=0,1, \ldots, p-1)$. In particular, we find $e_{1} e_{p-1}=f_{1} f_{p-1} / c^{p}$. Since $f_{1}, f_{p-1}$, and $c^{p} \in \bar{L}^{p}$, this shows $e_{1} e_{p-1} \in \bar{L}^{p}$. Now from (6), it is easy to see that

$$
\begin{aligned}
e_{1} & =-\left[\left(x_{1}^{p}-z^{p}\right) x_{2}^{p-1}+\left(x_{2}^{p}-z^{p}\right) x_{1}^{p-1}\right], \\
e_{p-1} & =\left[\left(x_{1}^{p}-z^{p}\right) x_{2}+\left(x_{2}^{p}-z^{p}\right) x_{1}\right] .
\end{aligned}
$$

Multiplying out, we find $x_{2} x_{2}^{p-1}+x_{2} x_{1}^{p-1} \in \bar{L}^{p}={\overline{F_{0}}}^{p}\left(x_{1}^{p}, x_{2}^{p}, z^{p}\right)$. Since $p>2$ (so $x_{1} x_{2}^{p-1}+x_{2} x_{1}^{p-1} \neq 0$ ), this is clearly impossible.

Case $2(p=2)$. Assume that

$$
\begin{aligned}
A_{n} \otimes_{F} E_{n-2} \sim & \left(y^{1 / 2^{n-2}}, b_{1} ; 2^{2}, E_{n-2}, \omega_{2}\right) \\
& \otimes E_{n-2}\left(b_{2}, \phi_{n} ; 2, E_{n-2},-1\right),
\end{aligned}
$$

for some $b_{1}$ and $b_{2} \in E_{n-2}^{*}$. Then, letting $z=y^{1 / 2^{n}}$ and $E_{n}=E(z)$, we find, exactly as in the $p$ odd case, that $z / b=N(u)$ for some $b \in E_{n-2}^{*}$ and $u \in E_{n}\left(\sqrt{\phi_{n}}\right)$, where $N$ denotes the norm from $E_{n}\left(\sqrt{\phi_{n}}\right)$ to $E_{n}$. Letting $\chi=h_{0}\left(x_{1}, x_{2}, z\right)$, assuming $F_{0}$ is algebraically closed, and considering the standard extension of any 2-adic valuation on $F_{0}$ to $F_{0}\left(x_{1}, x_{2}, \ldots, z\right)$, we find, just as in the $p$ odd case that for some $b_{0} \in \overline{F_{0}}\left(x_{1}, x_{2}, z^{4}\right)$ and $u_{0} \in \overline{F_{0}}\left(x_{1}, x_{2}, z\right)(\sqrt{\chi})$,

$$
z b_{0}=u_{0}^{2} \text {. }
$$

We will show that (19) is impossible.

Write $L$ for the field $\overline{F_{0}}\left(x_{1}, x_{2}, z\right), L_{1}$ for the field $\overline{F_{0}}\left(x_{1}, x_{2}, z^{2}\right)$, and $L_{2}$ for the field $\overline{F_{0}}\left(x_{1}, x_{2}, z^{4}\right)$. Assume that (19) holds for some $b_{0} \in L_{2}$ and $u_{0} \in L(\sqrt{\chi})$. By considering the $z$-adic valuation on $L$ and noting that $b_{0} \in L_{2}$, it is easy to see that $z b_{0} \notin L^{2}$. Hence, 
$z b_{0}=u_{0}^{2}$, then $L(\sqrt{\chi})=L\left(\sqrt{z b_{0}}\right)$. From this, as well as the definition of $h_{0}$ in (7), it follows that

$$
z\left(\left(x_{1}^{2}+z^{2}\right) x_{2}+\left(x_{2}^{2}+z^{2}\right) x_{1}\right)=f_{0}^{2}+f_{1}^{2} z b_{0},
$$

for some $f_{0}$ and $f_{1} \in L$. Since 1 and $z$ form a basis for $L$ as an $L_{1}$ vector space, and since $f_{0}^{2}, f_{1}^{2},\left(x_{1}^{2}+z^{2}\right) x_{2}+\left(x_{2}^{2}+z^{2}\right) x_{1}$, and $b_{0}$ are all in $L_{1}$, we find

$$
\left(x_{1}^{2}+z^{2}\right) x_{2}+\left(x_{2}^{2}+z^{2}\right) x_{1}=f_{1}^{2} b_{0} .
$$

We write this as

$$
\frac{x_{1}^{2} x_{2}+x_{2}^{2} x_{1}}{b_{0}}+\frac{z^{2}\left(x_{2}+x_{1}\right)}{b_{0}}=f_{1}^{2} .
$$

Now $f_{1}^{2} \in L^{2}=L_{1}^{2}\left(z^{2}\right)$. Thus $f_{1}^{2}=g_{0}^{2}+g_{1}^{2} z^{2}$ for some $g_{0}$ and $g_{1} \in L_{1}$. Substituting this in (20), we find

$$
\frac{x_{1}^{2} x_{2}+x_{2}^{2} x_{1}}{b_{0}}+\frac{z^{2}\left(x_{2}+x_{1}\right)}{b_{0}}=g_{0}^{2}+g_{1}^{2} z^{2} .
$$

Now $x_{1}^{2} x_{2}+x_{2}^{2} x_{1}, x_{2}+x_{1}$, and $b_{0}$ (note!) are all in $L_{2}$. Moreover, $L_{1}^{2} \subset L_{2}$. Since 1 and $z^{2}$ form a basis of $L_{1}$ as an $L_{2}$ vector space, we find on viewing (21) as an equation in $L_{1}$ that

$$
\frac{x_{1}^{2} x_{2}+x_{2}^{2} x_{1}}{b_{0}}=g_{0}^{2}
$$

and

$$
\frac{x_{2}+x_{1}}{b_{0}}=g_{1}^{2} \text {. }
$$

Dividing, we find $x_{1} x_{2}=\left(g_{0} / g_{1}\right)^{2}$ for some $g_{0}$ and $g_{1} \in L_{1}$. But $x_{1} x_{2}$ is clearly not a square in $L_{1}$, and we are done.

\section{REFERENCES}

[ART] S. A. Amitsur, L. H. Rowen and J.-P. Tignol, Division algebras of degree 4 and 8 with involution, Israel J. Math., 33 (1979), 133-148.

[B] N. Bourbaki, Algèbre Commutative, Chapitre VI, Hermann, Paris, 1961.

[D] P. K, Draxl, Skew fields, London Math. Soc. Lecture Note Series, Vol. 81, Cambridge Univ. Press, Cambridge, 1983.

[E] O. Endler, Valuation Theory, Springer-Verlag, New York, 1972.

[J] B. Jacob, Indecomposable division algebras of prime exponent, J. Reine Angew. Math., 413 (1991), 181-197.

[JW] B. Jacob and A. Wadsworth, Division algebras over henselian fields, J. Algebra, 128 (1990), 126-179. 
[O] A. Ostrowski, Untersuchungen zur arithmetischen Theorie der Körper, Math. Z., (1934), 269-404.

[R] L. H. Rowen, private communication.

[RT] L. H. Rowen and J.-P. Tignol, in preparation.

[Se1] B. A. Sethuraman, Constructions of valued division algebras with applications to indecomposability and elementary abelian crossed products, Doctoral Dissertation, University of California, San Diego, 1991.

[Se2] - Indecomposable division algebras, Proc. Amer. Math. Soc., 114 (1992), 661-665.

[T1] J.-P. Tignol, Produits croisés abéliens, J. Algebra, 70 (1980), 420-436.

[T2] _ Algèbres indécomposables d'exposant premier, Adv. in Math., 65 (1987), 205-228.

[T3] Central simple algebras with involution, in Ring Theory, Proceedings of the 1978 Antwerp Conference, F. Van Oystaeyen, ed., Marcel Dekker, New York, (1979), 279-285.

Received February 10, 1992.

California State University, Northridge

NORTHRIDGE, CA 91330 



\title{
PACIFIC JOURNAL OF MATHEMATICS
}

\author{
Founded by
}

\author{
E. F. BECKENBACH (1906-1982) F. Wolf (1904-1989)
}

\section{EDITORS}

Sun-Yung A. Chang

(Managing Editor)

University of California

Los Angeles, CA 90024-1555

chang@math.ucla.edu

\section{F. Michael Christ}

University of California

Los Angeles, CA 90024-1555

christ@math.ucla.edu

Herbert Clemens

University of Utah

Salt Lake City, UT 84112

clemens@math.utah.edu
THOMAS ENRIGHT

University of California, San Diego

La Jolla, CA 92093

tenright@ucsd.edu

Nicholas ERCOLANI

University of Arizona

Tucson, AZ 85721

ercolani@math.arizona.edu

R. FINN

Stanford University

Stanford, CA 94305

finn@gauss.stanford.edu

VAUGHAN F. R. JONES

University of California

Berkeley, CA 94720

vfr@math.berkeley.edu
SteVen KerCKHofF

Stanford University

Stanford, CA 94305

spk@gauss.stanford.edu

MARTIN ScharLemanN

University of California

Santa Barbara, CA 93106

mgscharl@math.ucsb.edu

Harold STARK

University of California, San Diego

La Jolla, CA 92093

V. S. VARADARAJAN

University of California

Los Angeles, CA 90024-1555

vsv@math.ucla.edu

\section{SUPPORTING INSTITUTIONS}

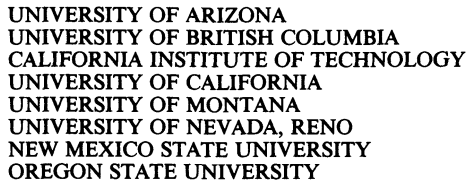

The Supporting Institutions listed above contribute to the cost of publication of this Journal, but they are not owners or publishers and have no responsibility for its content or policies.

Mathematical papers intended for publication in the Pacific Journal of Mathematics should be in typed form or offset-reproduced (not dittoed), double spaced with large margins. Please do not use built up fractions in the text of the manuscript. However, you may use them in the displayed equations. Underline Greek letters in red, German in green, and script in blue. The first paragraph must be capable of being used separately as a synopsis of the entire paper. In particular it should contain no bibliographic references. Please propose a heading for the odd numbered pages of less than 35 characters. Manuscripts, in triplicate, may be sent to any one of the editors. Please classify according to the 1991 Mathematics Subject Classification scheme which can be found in the December index volumes of Mathematical Reviews. Supply name and address of author to whom proofs should be sent. All other communications should be addressed to the managing editor, or Julie Honig, University of California, Los Angeles, California 90024-1555.

There are page-charges associated with articles appearing in the Pacific Journal of Mathematics. These charges are expected to be paid by the author's University, Government Agency or Company. If the author or authors do not have access to such Institutional support these charges are waived. Single authors will receive 75 free reprints; joint authors will receive a total of 100 free reprints. Additional copies may be obtained at cost in multiples of 50 .

The Pacific Journal of Mathematics (ISSN 0030-8730) is published monthly except for July and August. Regular subscription rate: $\$ 215.00$ a year (10 issues). Special rate: $\$ 107.50$ a year to individual members of supporting institutions.

Subscriptions, orders for numbers issued in the last three calendar years, and changes of address should be sent to Pacific Journal of Mathematics, P.O. Box 4163, Berkeley, CA 94704-0163, U.S.A. Old back numbers obtainable from Kraus Periodicals Co., Route 100, Millwood, NY 10546.

The Pacific Journal of Mathematics at University of California, c/o Department of Mathematics, 981 Evans Hall, Berkeley, CA 94720 (ISSN 0030-8730) is published monthly except for July and August. Second-class postage paid at Berkeley, CA 94704, and additional mailing offices. POSTMASTER: send address changes to Pacific Journal of Mathematics, P.O. Box 4163, Berkeley, CA 94704-0163.

PUBLISHED BY PACIFIC JOURNAL OF MATHEMATICS at University of California, Berkeley, CA 94720, A NON-PROFIT CORPORATION

This publication was typeset using $\mathcal{A} \mathcal{M} S-\mathrm{T}_{\mathrm{E}} \mathrm{X}$, the American Mathematical Society's $\mathrm{T}_{\mathrm{E}} \mathrm{X}$ macro system. Copyright (c) 1994 by Pacific Journal of Mathematics 


\section{PACIFIC JOURNAL OF MATHEMATICS}

Volume $162 \quad$ No. $2 \quad$ February 1994

On the existence of convex classical solutions to multilayer fluid

201 problems in arbitrary space dimensions

ANDREW FRENCH ACKER

Extremal functions and the Chang-Marshall inequality

VALENTIN V. ANDREEV and ALEC LANE MATHESON

Productive polynomials

RICHARD ARENS

On factor representations of discrete rational nilpotent groups and the 261 Plancherel formula

LAWRENCE JAY CORWIN and CAROLYN PFEFFER JOHNSTON

Commutants of Toeplitz operators on the Bergman space

ZELJKO CUCKOVIC

When $L^{1}$ of a vector measure is an AL-space

GUILlERMO P. CURBERA

A convexity theorem for semisimple symmetric spaces

305

KARL-HERMANN NEEB

Ideals of finite codimension in free algebras and the fc-localization

AMNON RosenmanN and ShMUEL Rosset

Dec groups for arbitrarily high exponents

BHARATH Al SETHURAMAN

Errata to: "The set of primes dividing the Lucas numbers has density $2 / 3$ "

JEFFREY C. LAGARIAS 